\title{
A Search for Planetary Nebulae around Hot White Dwarfs
}

\author{
K. Werner ${ }^{1}$, K. Bagschik ${ }^{2}$, T. Rauch ${ }^{1}$ and R. Napiwotzki ${ }^{3}$ \\ ${ }^{1}$ Institut für Astronomie und Astrophysik, Universität Tübingen, Germany; \\ ${ }^{2}$ Sternwarte Bonn, Germany; ${ }^{3}$ Dr.-Remeis-Sternwarte Bamberg, Germany
}

Time scales for post-AGB stellar evolution are still unclear. In order to study them it is useful to look for old PNe and compare nebula expansion and stellar evolutionary ages. We have selected very hot members from different white dwarf sub-classes. Many of them are objects recently discovered in the Hamburg-Schmidt Survey (Hagen et al. 1995). The sample includes three peculiar objects whose spectra show signatures of an extremely hot wind $\left(\approx 10^{6} \mathrm{~K}\right)$, namely absorption lines of ultra-high ionized metals, e.g. O VIII (Werner et al. 1995). A complete list is given in the table below. The search was performed by direct $\mathrm{H} \alpha$ narrow band imaging using a wide angle $\left(20^{\prime} \times 20^{\prime}\right)$ CCD camera (WWFPP) attached to the Calar Alto $1.23 \mathrm{~m}$ telescope (28.9.-3.10.1995).

The search was entirely negative - with one possible exception: The DO white dwarf PG 0109+111. An asymmetric nebulosity extends about 5' from the star to the southwest, and there are hints that it extends even further out. No WD central star of type DO (i.e. pure helium) is known, with the possible exception of PG 0108+101 (Reynolds 1987), but our image centered on this star does not show any nebulosity. If our detection of an emission nebula around PG 0109+111 is in fact the first PN around a DO white dwarf needs to be confirmed by detailed spectroscopic studies. PG 0109+111 is the most massive DO white dwarf $\left(\mathrm{M}=0.74 \mathrm{M}_{\odot}\right)$ and among the hottest DOs known $\left(\mathrm{T}_{\text {eff }}=110000 \mathrm{~K}, \log \mathrm{g}=8.0\right.$, $\mathrm{d}=280 \mathrm{pc}$; Dreizler \& Werner 1996). Assuming an expansion velocity of $20 \mathrm{~km} / \mathrm{sec}$, we arrive at a linear radius of $0.4 \mathrm{pc}$ and an expansion age of 20000 years. This contrasts with the post-AGB age of the white dwarf which exceeds 100000 years (Blöcker 1995).

\begin{tabular}{llll} 
spectral type & star & & \\
\hline DA & HS 0615+6535 & HS 2246+0640 & \\
DAO & HS 0231+0505 & HS 2033+0507 & \\
DO & KPD 0005+5106 & PG 0038+199 & PG 0109+111 \\
& PG 0046+0746 & PG 0108+101 & PG 0237+116 \\
DO ultrahigh-ionisation & HS 0158+2335 & HS 0713+3958 & HS 2027+0651 \\
PG 1159 & PG 0122+200 & HS 0444+0453 & HS 2324+3944
\end{tabular}

\section{REFERENCES}

Blöcker, T., 1995, A\&A, 299, 755

Dreizler, S., Werner, K. 1996, A\&A, 314, 217

Hagen, H.-J., Groote, D., Engels, D., Reimers, D. 1995, A\&AS, 111, 195

Reynolds, R.J., 1987, ApJ, 315, 234

Werner, K., et al., 1995, A\&A, 293, L75 

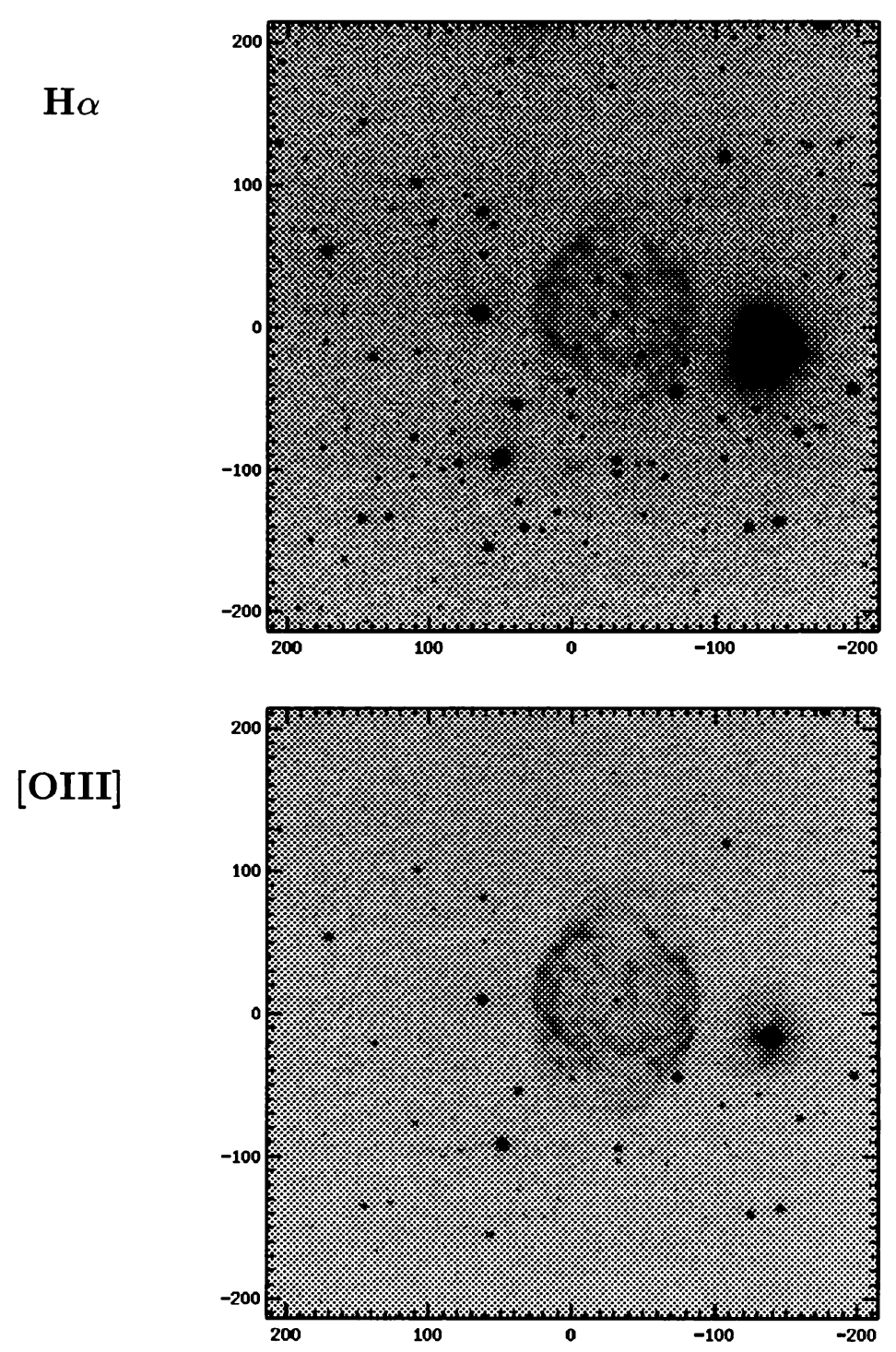

A $72 \quad 059.7-18.7$

From: "The IAC Morphological Catalog of Northern Galactic Planetary Nebulae", A. Manchado, M.A. Guerrero, L. Stanghellini, M. Serre-Ricart.

courtesy: A. Manchado 